\title{
SOCIAL ROBOTS: CHALLENGES FOR MACHINE INTELLIGENCE
}

Domain outline, main trends and issues

\author{
Raja Chatila, Georges Giralt \\ LAAS-CNRS, Toulouse, France
}

Front-line current developments in Robotics concern a vast spectrum of domains where close human-robot interaction plays a paramount role. These domains encompass Service (public-oriented, tour guide,...), Assistive (medical care, elderly care-taking, physically impaired,...) and Personal Robots (household, companions, entertainment,...).

Usually referred as Human Friendly Robotics and Human Centered Robotics, all those application cases emphasize the crucial aspect, well captured by Social Robots, of Human-Robot interaction issues where humans are most often non-expert users and by-standers. The very nature of this interaction brings to the forefront machine-intelligence central research issues of advanced Cognitive Robotics with key themes blending a large range of topics from embodiment and appearance to open-ended learning and decisional autonomy.

Hence, we have to confront a most highly challenging research framework encompassing a large set of technical themes in the demanding human-centered application perspective:

i) Multimodal communication: bi-lateral, appearance, context, behavior,...

ii) Perception: active, semantics, gestures, person identification,...

iii) Robot Motion: proximity with humans, cooperative tasks,...

iv) Learning: open-ended modalities, skill acquisition, understanding the universe of action, user tuning,... 
v) Autonomous decision: intentionality, planning, task reasoning, cooperative activities,...

vi) Dependability: safety, reliability, communication and operating robustness,...

The nine papers presented address these key themes bringing in results and on-going developments with a particular mention to Japan's two largescale programs on Humanoïd Robots and the European IST FET project COGNIRON.

The organization in three sessions, Overview and Challenges, Human Robot Interaction, and Learning and Cognition, aims to provide room for in depth thematic discussion with a concluding final panel stressing critical issues related to human-robot interaction and robot dependability, with machines endowed with salient features in the areas of decisional autonomy and open-ended learning. 